Irène Mestre

\title{
1 Approches environnementales et gestion des pâturages :
}

\section{le cas du Kirghizstan}

\section{Environmental approaches and pasture management. The case of Kyrgyzstan}

Les politiques de décentralisation de la gestion des ressources naturelles ont été promues par les organisations internationales, comme moyen pour lutter contre la pauvreté, garantir l'utilisation durable des ressources et s'assurer du bon usage des fonds accordés aux gouvernements. Les résultats n'ont cependant pas toujours été à la hauteur des espérances [Agrawal et Ostrom 2007]. La décentralisation a fait l'objet de nombreuses études et autant de débats. A. Agrawal et J. Ribot [1999] l'analysent à partir des effets qu'elle induit chez les acteurs (organismes ou personnes) directement concernés, notamment l'évolution de leurs prérogatives et de leurs responsabilités ${ }^{1}$. Ils les répartissent en trois groupes : les gouvernements centraux, les organisations internationales et les communautés locales, qui comprennent les usagers des ressources naturelles et les municipalités. Or aucun ne dispose à la fois des moyens financiers et de la capacité d'influence sur les autres institutions pour mettre seul en place ces politiques. L'enjeu crucial de la décentralisation est donc de parvenir à obtenir le soutien d'au moins deux groupes d'acteurs [Agrawal et Ostrom op. cit.]. À travers un cas d'étude sur la réforme de la gestion des pâturages au Kirghizstan, nous explorerons comment les contradictions dans les attitudes environnementales portées par les différents groupes freinent l'élaboration d'objectifs communs pour la décentralisation de la gestion des ressources naturelles. Nous verrons aussi que ces approches divergentes peuvent évoluer et se mélanger.

Pâturages d'été Čong taš, village de Žergetal, août 2014.

1 A. Agrawal et J. Ribot parlent d'« accountability». 


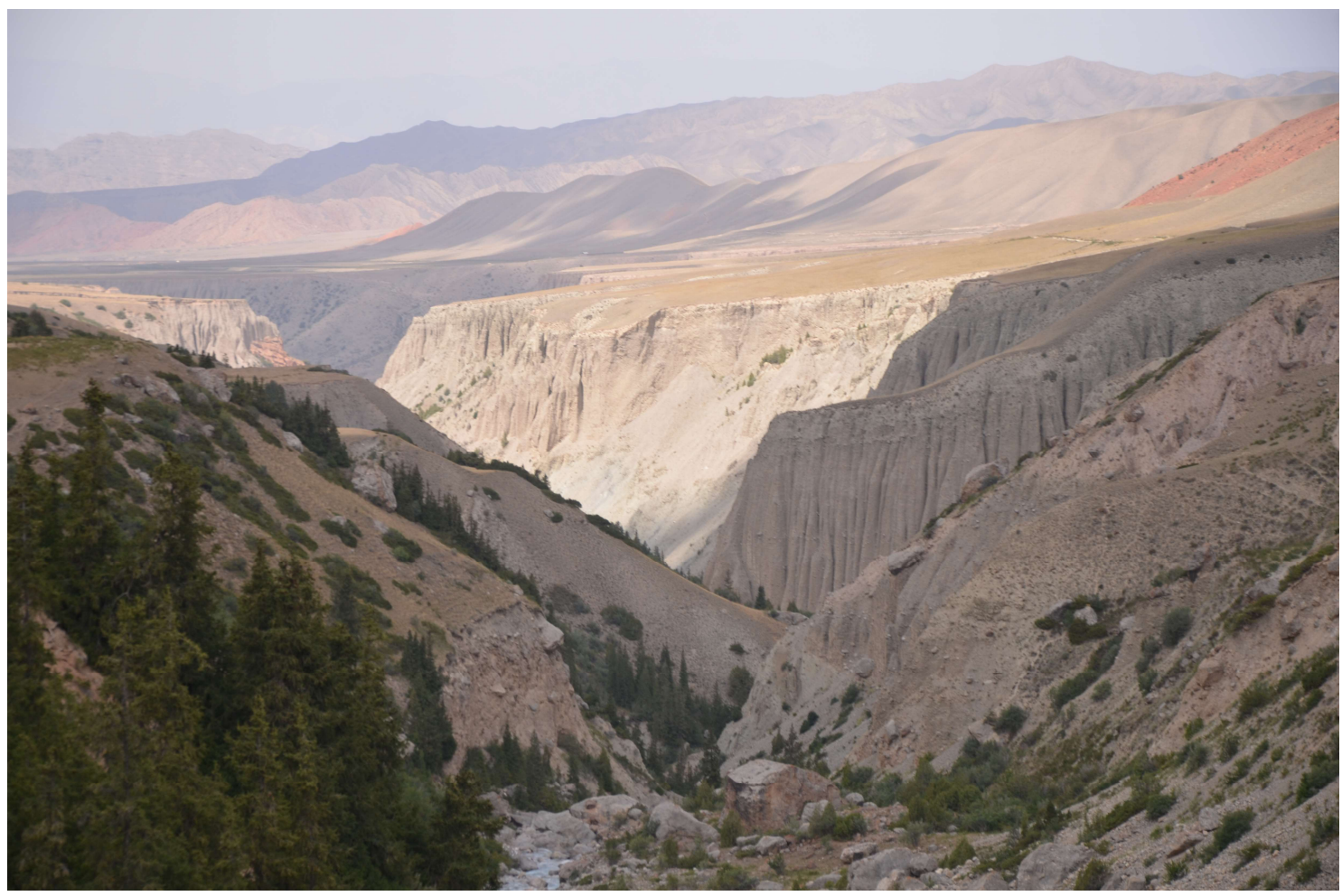

Le pâturage représenté sur cette photographie au premier plan est divisé en deux terrains adjacents, I'un géré par l'UGF (unité de gestion forestière), l'autre par l'AUP (association d'usagers des pâturages), sans matérialisation des limites.

Photo : I. Mestre

En 2009, est adoptée au Kirghizstan une loi sur les pâturages, qui va profondément en réformer le mode d'allocation et la responsabilité des infrastructures d'accès, jusque-là répartis entre les régions, les districts et les communes. Dorénavant ces missions sont confiées à des associations d'usagers des pâturages (AUP) à l'échelle municipale.

Cette loi a été fortement soutenue par les organisations internationales, notamment la Banque Mondiale. Il s'appuie en grande partie sur le principe selon lequel les communautés locales peuvent gérer de manière durable une ressource commune quand certaines conditions sont réunies [Ostrom 1990].

Cependant, cette politique de décentralisation n'a pas été menée à son terme, car plus d'un million d'hectares de pâturages demeurent gérés par l'administration des unités de gestion forestière (UGF), responsables de la valorisation et de la protection des terres du Fonds forestier d'État, sous l'autorité de l'Agence de protection des écosystèmes ${ }^{2}$ [Dörre 2015]. La part importante de pâturages dans les UGF s'explique par un transfert effectué par les municipalités rurales après l'indépendance afin de limiter les paiements de l'impôt foncier. Cette réforme, bien que s'inscrivant dans une tendance amorcée dès 1991 [Crewett 2016], marque un tournant dans la construction des approches de l'environnement auparavant très cloisonnées. En effet, pour lancer la gestion communautaire des pâturages, elle a mis en relation des partenaires ayant des conceptions et des

\footnotetext{
${ }^{2}$ Agence de protection des écosystèmes, Raspredelenie obŝej ploŝadi hozâjstv po kategoriâm zemel' [Répartition des terres par catégories] (document non publié), 2006.
} 
stratégies différentes. Le gouvernement central non seulement risquait d'imposer des règles peu adaptées aux spécificités locales [Dörre op. cit. ; Shigaeva et al. 2016], mais aussi il n'y avait pas d'unanimité en son sein : le ministère de l'Agriculture ${ }^{3}$ (avec son Département des pâturages) prône I'utilisation maximale des ressources, tandis que l'Agence de protection des écosystèmes, qui définit en grande partie les actions des UGF, défend, comme son nom l'indique, la conservation des milieux naturels.

Les organisations internationales présentes dans le pays accordent des financements au gouvernement et délèguent la mise en œuvre de projets pilotes à des organisations non gouvernementales (ONG). Enfin, à l'échelle locale, les 454 communes rurales confient la gestion de leurs pâturages aux AUP, mais elles valident leur plan annuel ainsi que leur budget prévisionnel.

Nous nous interrogeons d'abord sur les approches de l'environnement mobilisées par les différents acteurs dans la mise en place du mode de gestion communautaire des pâturages, puis sur l'appropriation de ces approches par les AUP pour définir les modalités d'utilisation des pâturages.

\subsection{Une méthodologie d'enquête pour explorer les interactions entre échelon national et local}

Le Kirghizstan est un pays de montagnes dont $95 \%$ du territoire est situé au-delà de $1000 \mathrm{~m}$ d'altitude. Les pâturages couvrent $89 \%$ de la surface agricole ${ }^{4}$ [Fitzherbert 2006]. Les habitants des zones rurales (64 \% de la population ${ }^{5}$ ) dépendent doublement de l'élevage agropastoral. En effet, il leur permet de consommer de la viande et des laitages, d'avoir des liquidités, grâce à la vente de leur production, et d'épargner sous forme de bêtes sur pied ${ }^{6}$. Le bétail, nourri majoritairement avec du fourrage pendant l'hiver, est déplacé dès le printemps de manière à exploiter le couvert végétal en fonction de l'altitude et de l'exposition des pâturages [Žaparov 2010]. Les activités d'élevage dominent dans les montagnes et la production végétale dans les vallées du Ferghana et de la Chouï.

La zone qui nous intéresse dans cette étude comprend trois municipalités rurales situées dans la région de Naryn et une dans celle de Djalal-Abad (voir carte et tableau 1).

3 La dénomination du ministère en charge de l'agriculture après avoir changé plusieurs fois est devenue " ministère de l'Agriculture et de la Bonification » en 2014. Afin de faciliter la lecture nous l'appelons ministère de l'Agriculture.

${ }^{4}$ A. R. Fitzherbert, "Kyrgyzstan country pasture/forage resource profile », Working Paper, Food and Agriculture Organization of the United Nations, 2006.

5 World Development Indicators, Rural population in percentage of the total population, World Bank, 2017 (<http://data.worldbank.org/indicator/SP.RUR.TOTL.ZS?locations=KG>).

6 A. Undeland et A. M. Mitchell, « Kyrgyz Republic - Communities forests and pastures", working paper, Banque mondiale, 2015

(<http://documents.worldbank.org/curated/en/550371468263989781/Kyrgyz-RepublicCommunities-forests-and-pastures $>$ ). 


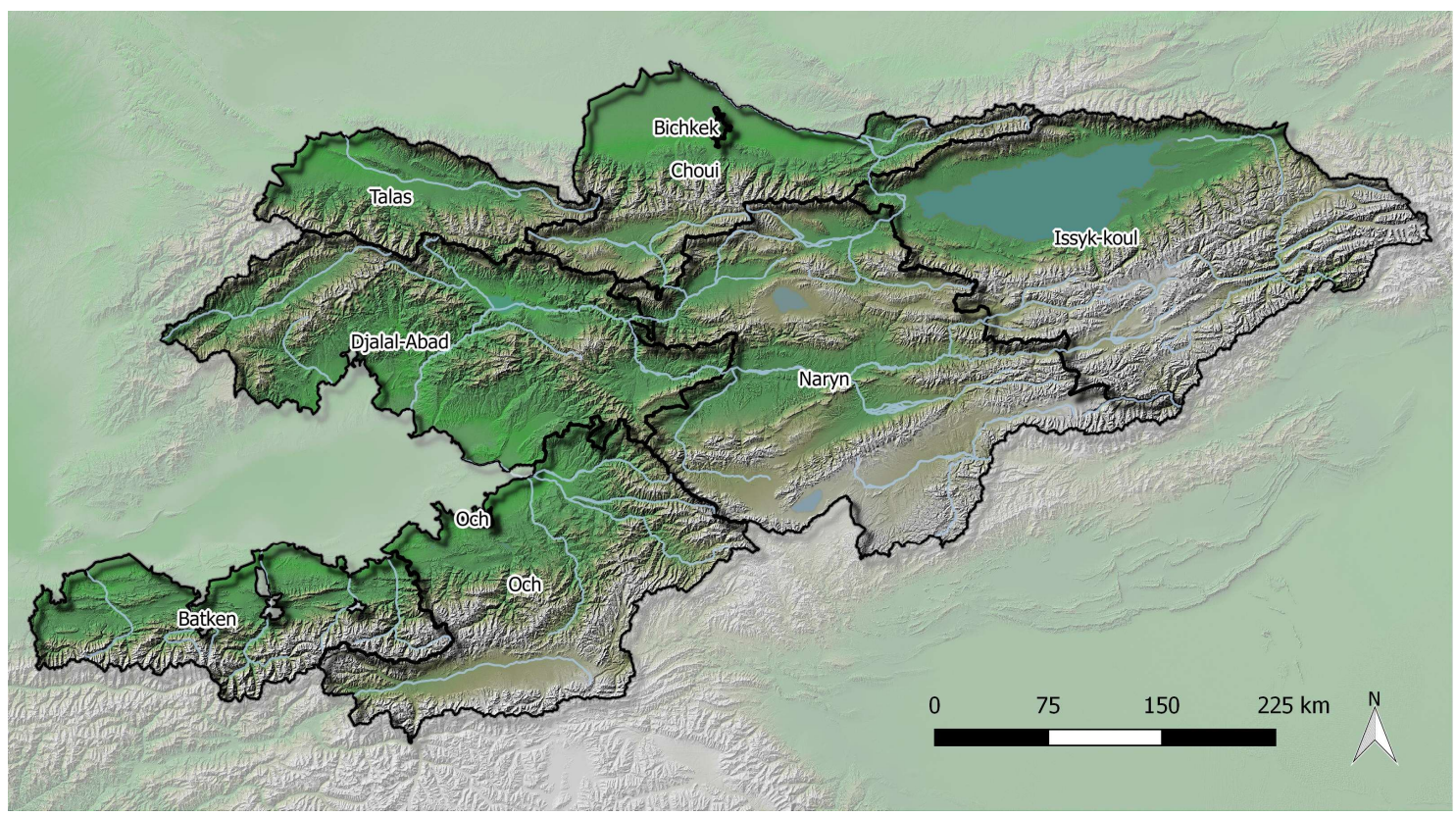

Carte des régions et principales villes du Kirghizstan

Carte : I. Mestre.

L'approche adoptée s'appuie sur celle de H. Gumuchian, É. Grasset, R. Lajarge et E. Roux [2003], qui souligne l'importance des acteurs de terrain dans la recomposition des territoires. La collecte de données s'est déroulée entre 2011 et 2015, dans les municipalités et dans la capitale pour explorer le rôle des acteurs de l'échelle internationale et nationale. Le corpus utilisé rassemble des entretiens semi-directifs, des observations ${ }^{7}$, de la cartographie, de la littérature " grise » et académique.

7 Les entretiens avec les acteurs internationaux et nationaux ont été menés en anglais et en russe sur 21 mois. L'enquête dans les municipalités et auprès des familles s'est déroulée pendant cinq mois à Altyn, Emgek-Talaa et Žergetal, à différents moments du calendrier agricole, dans les hameaux et sur les estives ; à Terek-Say, pendant un seul séjour. Les entretiens ont été conduits en russe et, avec l'assistance d'un traducteur, en kirghize. 


\begin{tabular}{|l|c|c|c|c|}
\hline $\begin{array}{l}\text { Région } \\
\text { (oblast) }\end{array}$ & $\begin{array}{c}\text { Municipalités } \\
\text { rurales } \\
\text { (ajyl ajmak) }\end{array}$ & Population & $\begin{array}{c}\text { Surface } \\
\text { de pâturage } \\
\text { (en ha) }\end{array}$ & $\begin{array}{c}\text { Nombre de tête } \\
\text { de bétail } \\
\text { (en unité gros bétail)' }\end{array}$ \\
\hline NARYN & Altyn & 3500 & 28000 & 7800 \\
\hline NARYN & Emgek Talaa & 2413 & 22289 & 3158 \\
\hline NARYN & Žergetal & 2298 & 43514 & 4349 \\
\hline DJALAL-ABAD & Terek-Saj & 3490 & 10990 & 3757 \\
\hline
\end{tabular}

1. D'après l'arrêté sur les modalités de l'établissement du paiement de l'utilisation des pâturages n³86 (19/06/2009), une tête de grand bétail (bovin, équin, camelin) adulte équivaut à une unité gros bétail (UGB) ; une tête de petit bétail (caprin, ovin) à 0,2 UGB.

Tableau 1. Caractéristiques des municipalités rurales sélectionnées pour les cas d'étude (données locales, 2011)

\subsection{Les associations d'usagers des pâturages dans un système centralisé et fragmenté}

Le ministère de l'Agriculture et son Département des pâturages sont, en grande partie, les héritiers des institutions soviétiques. Dans toute l'Asie centrale, l'expansion des activités agricoles était hautement politisée et considérée comme un pilier du développement de la région [Wolfgramm et al. 2015]. À partir des années 1960, le concept d'utilisation rationnelle des ressources naturelles ${ }^{8}$ a permis de concilier l'intensification de la production agricole et un certain niveau de protection des ressources naturelles. Ses applications sont, par exemple, le développement de pâturages artificiels, la pulvérisation d'herbicides pour les plantes non comestibles pour le bétail ou l'extension des infrastructures pastorales ${ }^{9}$.

Les UGF et l'Agence de protection des écosystèmes sont, de leur côté, les héritières de la conception soviétique de la préservation des espaces naturels. Les premières réserves (zapovedniki) dédiées à la protection et l'étude de la nature ont été inaugurées à l'époque tsariste et se sont multipliées en URSS dans les années 1920 et 1930 [Weiner 1982]. À la fin de la période soviétique, on dénombrait quatre réserves naturelles au Kirghizstan et six nouvelles ont été créées depuis l'indépendance. Elles se consacrent principalement à la protection des écosystèmes forestiers, qui ne couvrent que 5,6\% du territoire ${ }^{10}$. Le secteur forestier est tourné vers des fonctions de conservation et de recherche plutôt que vers l'exploitation industrielle [Schmidt et Doerre 2011].

La période suivant l'indépendance a été marquée par deux révolutions, en 2005 et en 2010, et par l'arrivée d'organisations internationales. Dans le domaine agricole et environnemental, l'Agence de coopération internationale allemande pour le développement, l'agence des États-Unis pour le développement international, la Banque mondiale, le Fonds international de développement

\footnotetext{
${ }^{8}$ Définie dans la norme gouvernementale GOST standard 26640-85.

9 Voir l'article d'l. Ohayon dans ce même numéro.

${ }^{10}$ Comité national des statistiques, Okružaûŝâ̂ sreda v Kyrgyzskoj Respublike 2011-2015

[L'Environnement dans la République kirghize 2011-2015], Bichkek, 2016.
} 
agricole des Nations unies, le Programme des Nations unies pour le développement et la Direction du développement et de la coopération de la Confédération suisse accompagnent l'entrée dans l'économie de marché en soutenant l'adoption de nouvelles politiques publiques. L'attention du gouvernement et des organisations internationales s'est d'abord portée sur la privatisation des terres arables, alors que les pâturages sont restés la propriété de l'État ${ }^{11}$. Les années 1990 sont celles où le modèle de production agricole s'est radicalement transformé avec le démantèlement des kolkhozes et des sovkhozes ainsi que l'apparition d'une multitude d'exploitations familiales, au nombre de 400794 en $2015^{12}$. Lors de la décennie suivante, de nouveaux acteurs gouvernementaux et non gouvernementaux sont apparus et des changements importants ont eu lieu dans le cadre légal de l'utilisation et de la gestion des pâturages (voir tableau 2).

En 2002, une loi permettant la location des pâturages a été adoptée. Elle instaurait de fait une privatisation des usages en excluant une partie des usagers et en garantissant des droits d'usage exclusifs [Crewett 2012]. Des baux pouvaient être conclus entre les particuliers et les administrations régionales pour les pâturages d'été, de district pour les pâturages d'automne et de printemps, et municipales pour ceux d'hiver. Les organisations internationales ont vu dans cette fragmentation de la gestion l'un des principaux facteurs de limitation des distances de transhumance et donc de la dégradation des pâturages ${ }^{13}$. Elles ont proposé de penser la question des ressources naturelles en termes de développement durable, en considérant les politiques de décentralisation initiées auparavant comme un remède contre les conséquences de la privatisation.

Les organisations internationales ont soutenu la préparation de la réforme des pâturages, dans le but de mettre en place une gestion durable de la ressource pastorale permettant de réduire la dégradation des pâturages et d'augmenter la productivité du bétail ${ }^{14}$ (voir tableau 2). Cette évolution du cadre légal se distingue des approches antérieures par l'égale importance attribuée à la croissance économique, la cohésion sociale et la protection de l'environnement. Les actions des organisations internationales se sont traduites par la création du Département des pâturages, qui a bénéficié de leur soutien pour préparer, puis mettre en place la réforme des pâturages, de l'Agence de développement et d'investissement pour les communautés chargées de réaliser les programmes financés, ainsi que par la création d'ONG.

Le Département des pâturages et les ONG ont joué un rôle important dans la mise en place de projets pilotes préfigurant la loi de 2009 , laquelle a fait l'objet de financements internationaux

${ }^{11}$ R. Mogilevskii, et al., Sheep meat production value chains in the Kyrgyz Republic and export capacity to the EAEU member states, Working paper series $n^{\circ} 36,2017$, Institute of Public Policy and Administration, Bichkek.

${ }^{12} \mathrm{Idem}$.

${ }^{13}$ B. Shamsiev (dir.), Kyrgyz Republic. Livestock Sector Review: Embracing the New Challenges, World Bank, 2006.

${ }^{14}$ Pasture Reform, Suggestions for improvements to pasture management in the Kyrgyz Republic, 2007 (<http://pdf.usaid.gov/pdf_docs/PNADN532.pdf>) et Land reform and market development project II, Final report, United States Agency for International Development, Bichkek, 2010 (<http://pdf.usaid.gov/pdf_docs/Pdacp456.pdf>). 
prévus pour atteindre au moins 61 millions de dollars en $2020^{15}$. À travers ses branches locales, ce Département apporte un soutien aux AUP en les aidant à élaborer et à réaliser leurs plans de gestion. Cependant, du fait de la faiblesse des moyens à disposition dans les branches locales et des distances importantes à parcourir pour aller dans les communes, leurs fonctions s'orientent surtout vers la collecte de données (budget et actions mises en place) pour le Département des pâturages. Elles font également office de relais entre les ONG et les AUP.

En 2004, avant les AUP, des associations d'usagers de l'eau d'irrigation ont été créées avec le soutien des organisations internationales. Leur bilan après plus de dix ans de fonctionnement montre la faiblesse de leurs capacités, en partie liée à l'absence d'engagement de l'État [Schmitt 2015].

Tableau 2. Chronologie des textes de loi et de leur incidence sur la gestion des pâturages

\begin{tabular}{|c|c|c|c|}
\hline Année & Textes adoptés & Nouveaux acteurs & $\begin{array}{l}\text { Effets sur l'utilisation } \\
\text { et la gestion des pâturages }\end{array}$ \\
\hline 2002 & $\begin{array}{l}\text { Loi sur « les } \\
\text { procédures pour la } \\
\text { location et l'usage des } \\
\text { pâturages » }\end{array}$ & & $\begin{array}{l}\text { Location aux usagers et possibilités } \\
\text { d'exclusion }\end{array}$ \\
\hline \multirow[t]{3}{*}{2003} & & $\begin{array}{l}\text { Département des pâturages (issu } \\
\text { de l'Institut républicain de } \\
\text { construction et d'amélioration } \\
\text { pastorale) }\end{array}$ & $\begin{array}{l}\text { Proposition de textes de loi sur les } \\
\text { pâturages, mise en place des réformes } \\
\text { adoptées }\end{array}$ \\
\hline & & $\begin{array}{l}\text { Agence de développement et } \\
\text { d'investissement pour les } \\
\text { communautés }\end{array}$ & $\begin{array}{l}\text { Structure mixte créée par décret } \\
\text { présidentiel, chargée de réaliser des projets } \\
\text { dans les zones rurales en coopération avec } \\
\text { les municipalités }\end{array}$ \\
\hline & & Rural Development Fund (ONG) & $\begin{array}{l}\text { Réintroduction des savoir-faire pré- } \\
\text { soviétiques liés aux ressources naturelles } \\
\text { pour développer les communautés rurales }\end{array}$ \\
\hline 2006 & & CAMP Alatoo (ONG) & $\begin{array}{l}\text { Développer les communautés rurales } \\
\text { grâce à une meilleure gestion des } \\
\text { ressources naturelles }\end{array}$ \\
\hline 2009 & $\begin{array}{l}\text { Loi sur « Sur les } \\
\text { pâturages » }\end{array}$ & & $\begin{array}{l}\text { Mise en place d'une gestion des pâturages } \\
\text { par des associations d'usagers à l'échelle de } \\
\text { la commune }\end{array}$ \\
\hline 2010 & & $\begin{array}{l}\text { Kyrgyz Association of Forest and } \\
\text { Land Users (ONG) }\end{array}$ & $\begin{array}{l}\text { Réseau d'échanges de bonnes pratiques } \\
\text { agronomiques, promotion de solutions } \\
\text { techniques et renforcement des capacités } \\
\text { institutionnelles des membres }\end{array}$ \\
\hline 2011 & $\begin{array}{l}\text { Amendement à la loi } \\
\text { «Sur les pâturages » }\end{array}$ & & $\begin{array}{l}\text { Renforcer la responsabilité et la } \\
\text { transparence des comités de pâturages } \\
\text { face aux membres des associations } \\
\text { d'usagers. }\end{array}$ \\
\hline 2013 & & $\begin{array}{l}\text { Fédération des associations des } \\
\text { usagers des pâturages (ONG) }\end{array}$ & $\begin{array}{l}\text { Renforcer l'échange d'expérience et les } \\
\text { actions conjointes entre les associations } \\
\text { d'usagers des pâturages et promouvoir } \\
\text { leurs intérêts à l'échelle nationale }\end{array}$ \\
\hline 2015 & & Muras Bašaty (ONG) & Développer les capacités des AUP \\
\hline
\end{tabular}


Les communes, constituées d'un ou de plusieurs hameaux, résultent de changements successifs du régime des collectivités locales. En 1996, les conseils ruraux ont été remplacés par les conseils municipaux. Les municipalités se sont formées sur les territoires des sovkhozes ou des kolkhozes [Pétric et al. 2004].

Les maires et les conseillers municipaux, responsables du bien-être des habitants, perçoivent les pâturages comme une ressource à exploiter pour permettre aux familles de vivre dignement en l'absence d'autres opportunités économiques. Comme le souligne A. Dörre [2015], la décentralisation de la responsabilité des pâturages à l'échelle municipale s'est traduite par une contraction des prérogatives de l'État, ce qui lui permettait de réduire ses dépenses, mais représentait pour les administrations locales un défi au vu des faibles moyens dont elles disposaient par rapport à leurs besoins.

Les AUP ont été créées en 2009 par la loi "Sur les pâturages », qui autorise les municipalités rurales à leur déléguer la gestion des pâturages et de leurs infrastructures. Le pays en compte $454^{16}$, en relation avec des acteurs de l'échelle nationale et locale (voir schéma). Cette réforme ne concerne pas les pâturages sous la responsabilité des UGF [Dörre op. cit.].

Schéma des interactions entre les associations d'usagers des pâturages (AUP) et les acteurs de l'échelle nationale et locale.

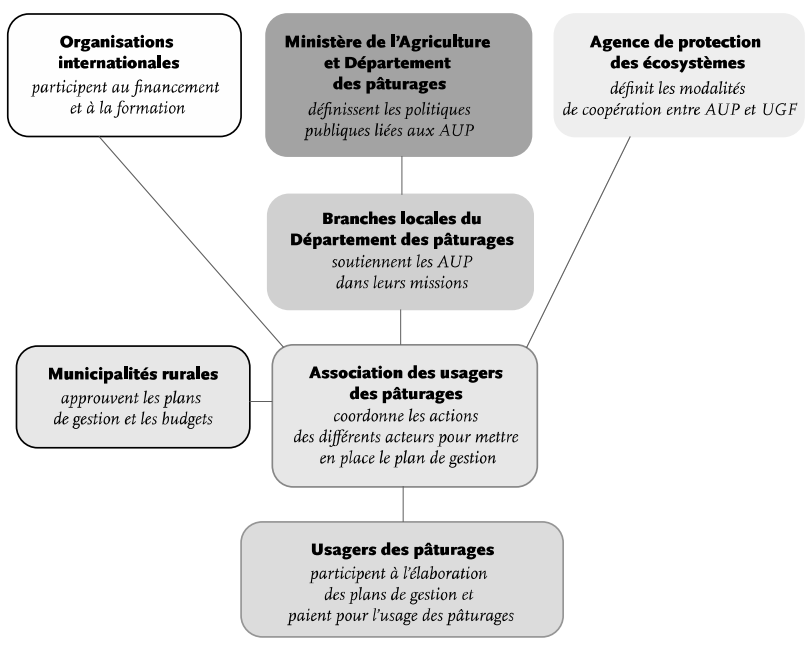

Les AUP regroupent toutes les personnes morales et physiques dont l'activité est liée aux pâturages sur le territoire d'une commune. Leurs membres doivent représenter les intérêts des agropasteurs et des autres usagers dans les décisions de gestion. Les premiers se divisent en deux catégories : les propriétaires de bétail et les bergers, à qui ils confient leurs troupeaux pour la montée en estive entre mai et octobre. Ceux-ci représentent moins de $20 \%$ des ménages dans la zone d'étude. Les

${ }^{16}$ A. Egemberdiev, Pastbiŝnaâ reforma v Kyrgyzstane: problemy i puti ih rešeniâ [La réforme pastorale au Kirghizistan: problèmes et perspectives, présentation], ministère de l'Agriculture du Kirghizistan, 2014. 
parcours peuvent se situer sur le territoire d'une commune (à Altyn et à Emgek-Talaa) ou plusieurs et traverser des UGF (à Terek-Saj et Žergetal).

Un nombre restreint d'usagers développe des activités non agropastorales comme l'accueil de touristes, la cueillette d'herbes aromatiques, médicinales ou des activités minières. Terek-Saj et Altyn sont des exceptions à l'échelle nationale avec plus de $50 \%$ des ménages qui combinent activités minières et agropastorales [Mestre 2017].

D'après la loi, tous les usagers participent à l'élection des membres de l'organe exécutif que constitue le comité des pâturages (CP) et de son directeur. Des conseillers municipaux peuvent également être membres du CP, mais sans devenir majoritaires. Le CP élabore un plan annuel d'utilisation et un plan de gestion quinquennal, qui doivent être approuvés par le conseil municipal. Son bilan d'activité doit être présenté à l'assemblée générale de l'AUP.

Les AUP sont financées par des organisations internationales et par le paiement pour l'utilisation des pâturages. Calculé par tête de bétail, son montant est fixé localement. En 2011 sur la zone d'étude, il variait de $0,15 \$$ à $0,45 \$$ pour une chèvre ou un mouton et de $0,80 \$$ à $1,35 \$$ pour une tête de gros bétail, respectivement à Altyn et à Terek-Saj.

Les dépenses des AUP comprennent les frais de fonctionnement, l'impôt foncier, les actions et mesures d'amélioration des pâturages et une contribution au budget de la municipalité dont le montant n'est pas déterminé par la loi. Entre 2010 et 2012, la part des subventions atteignait, dans les trois cas d'étude, jusqu'à $90 \%$ des revenus des AUP, puis elle s'est réduite jusqu'à 20-30\%, à mesure que les revenus liés au paiement pour l'usage des pâturages augmentaient. Les financements des bailleurs de fonds sont accompagnés de formations pour le renforcement des compétences des membres des CP et des AUP. Les mesures techniques, en particulier le suivi de la qualité des pâturages, pour lesquels le Département des pâturages n'a pas approuvé de guide méthodologique, sont rarement mises en place dans les quatre communes.

Malgré les dispositions de la loi, qui attribuent aux assemblées générales d'usagers un rôle majeur dans la prise de décision, ces réunions sont organisées de manière irrégulière, sans suivre les règles formelles et ne donnent pas d'orientation pour le CP. Celui-ci décide souvent seul des actions à mettre en place [Isaeva et Shigaeva 2017], mais il peut être destitué sous la pression des bergers, bergers-éleveurs ou des propriétaires de bétail [Mestre op. cit.].

\subsection{L'environnement peut-il être une compétence communautaire?}

Outre leur influence sur le ministère de l'Agriculture, les organisations internationales sont en contact avec les associations d'usagers des pâturages à travers les projets mis en place localement par des ONG qu'elles soutiennent. Les membres des AUP des quatre communes du terrain de recherche ont bénéficié de formations et de subventions des organisations internationales données par l'Agence de développement et d'investissements pour les communautés ${ }^{17}$. La réparation des voies d'accès est présentée comme le pilier de la gestion : elle permet, en effet, de mieux contrôler les déplacements vers les pâturages d'été ; bergers et propriétaires y voient aussi des réalisations concrètes qui justifient le règlement de leur cotisation.

\footnotetext{
${ }^{17}$ Au programme de ces formations entre 2009 et 2015: le fonctionnement administratif et les droits des AUP, les moyens pour inciter les usagers à régler leur cotisation pour l'usage des pâturages, la collecte de ces cotisations ou le contrôle des dates de transhumance.
} 
Deux projets ont été mis en place dans la zone d'étude par la même ONG et financés par l'Agence allemande de coopération internationale. Le premier, en 2010, à Altyn, a vu le jour avant même l'adoption légale des AUP. II s'est traduit par des réunions avec les habitants, les conseillers municipaux et l'administration locale visant à préparer la création de l'AUP, des comités des pâturages, à élaborer un plan de gestion (dont le financement d'une route et d'un pont menant à des pâturages d'altitude) et à mettre en place des mesures de suivi de la qualité des pâturages. Ces dernières n'ont pas été poursuivies par l'AUP une fois le projet achevé. Le projet mis en place à Žergetal en 2015 portait sur le changement climatique et la gestion des conflits. Des séminaires ont été conduits, un support financier et technique apporté à la construction d'une portion de route vers des pâturages d'été. Les AUP de ces deux communes continuent de chercher des financements pour l'extension ou la rénovation de la voirie.

La priorité donnée aux infrastructures correspond à la politique d'aménagement du ministère de I'Agriculture ${ }^{18}$. Ainsi le Département des pâturages utilise comme indicateur de succès de la réforme le nombre de kilomètres de routes et de ponts réparés ou reconstruits ${ }^{19}$.

Après l'indépendance, l'expression soviétique " utilisation rationnelle " a progressivement été remplacée par celle de " gestion durable » portée par les organisations internationales, à tel point qu'aujourd'hui, on les utilise souvent de façon interchangeable [Shigaeva et al. 2013]. Alors que la première met l'accent sur l'intensification de l'exploitation, la seconde cherche à combiner croissance économique, justice sociale et protection de l'environnement pour répondre aux besoins actuels, sans remettre en cause la capacité des générations futures à répondre aux leurs. La confusion entre les deux s'est accentuée au fur et à mesure que des organisations internationales se sont emparées de la question environnementale. La notion de dégradation des pâturages, centrale dans l'adoption du cadre légal des AUP, est elle aussi diversement définie par les organisations internationales, les agences gouvernementales et les agropasteurs. L'absence d'approche unifiée de la dégradation est un frein important à l'évaluation de la qualité des pâturages [Levine et al. 2017].

Si la plupart des organisations internationales actuelles sont présentes dans le pays depuis I'indépendance, de nouvelles ONG ont été créées après 2000, chacune développant sa propre approche. Toutes ont en commun la lutte contre la dégradation des pâturages et la volonté de renforcer la gestion locale et son rôle transversal de coordination avec les autres institutions (municipalités ou UGF) et les usagers non agropastoraux. Par exemple, le programme des Nations unies pour le développement s'est focalisé sur les outils de gestion techniques pour l'allocation des pâturages tels que les systèmes d'information géographique ${ }^{20}$. La coopération allemande se concentre sur la prise en compte écosystémique et l'adaptation au changement climatique ${ }^{21}$. Quant aux agences de coopération étasuniennes et britanniques, elles ont conduit d'importants projets sur

${ }^{18}$ Ministère de l'Agriculture et de la Bonification de la République kirghize, Programma razvitiâ pastbiŝnogo hozâjstva Kyrgyzskoj Respubliki na 2012-2015 gody [Programme de développement de l'exploitation des pâturages 2012-2015], n89, adopté le 10/02/2012, Bichkek.

${ }^{19}$ A. Egemberdiev [op. cit.].

${ }^{20} \mathrm{~J}$.-J. Bellamy, Final Evaluation of the UNDP-Supported, GEF-financed project: Demonstrating sustainable Mountain Pasture Management in the Suusamyr Valley, Kyrgyzstan, programme des Nations unies pour le développement et Fonds pour l'environnement mondial, 2013.

21 Leurs projets liés aux pâturages sont « Ecosystem-based Adaptation to climate change in Central Asia » et «Community-based Walnut Forest and Pasture Management in Southern Kyrgyzstan ». 
la gestion des conflits liés aux ressources naturelles, perçus comme pouvant conduire à des nouvelles violences ${ }^{22}$.

Bien que partageant un même objectif de développement durable, les ONG et les organisations internationales proposent différentes méthodes de calcul de la capacité de charge du bétail sur les pâturages. Les tentatives informelles du comité de coordination de promouvoir une approche plus unifiée n'ont pas abouti. Les modes de calcul se différencient à plusieurs niveaux : (1) sur le facteur principal de l'évolution de la qualité et de la quantité de la biomasse, à savoir la variabilité interannuelle des precipitations ou l'utilisation à des fins d'élevage ou une combinaison de facteurs ${ }^{23}$; (2) sur les méthodes de collecte de données; et (3) sur les outils d'analyse des données. $\mathrm{Si}$ les grandes lignes des modes de calcul sont connues, un flou demeure sur les formules appliquées [Robinson 2016].

Cependant l'absence de coordination à l'échelle nationale contraste avec la volonté des AUP de joindre leurs forces. Dès 2013 et à leur propre initiative, l'AUP de Terek-Saj s'est organisée avec les autres AUP du district sous forme d'association, afin de coordonner leurs actions de gestion et d'avoir plus de poids face aux acteurs gouvernementaux. Les associations de districts cherchent notamment à faciliter l'organisation de parcours de transhumance entre les territoires de plusieurs communes.

À la fin de cette même année a été créée la fédération kirghize des associations des usagers des pâturages, dont les membres sont les AUP et des associations d'AUP, dans le but de protéger leurs intérêts au niveau national et de capitaliser leurs expériences. Depuis 2015, elle est dirigée par Abdymalyk Abdikaarovič Egemberdiev, l'ancien directeur du Département des pâturages qui a mené la réforme. Sa légitimité est incontestable auprès des AUP, des employés des branches locales du Département ainsi qu'auprès de nombreux politiciens, car il a surmonté les oppositions et assuré I'implication des organisations internationales. La fédération, qui réunissait 400 des 454 AUP en 2015, prône l'adoption d'un cadre légal plus contraignant pour réguler les relations avec les usagers non agropastoraux et élaborer des guides opérationnels sur l'encadrement des activités non liées à l'élevage prenant place sur les pâturages.

Les associations d'usagers des pâturages sont dans une position paradoxale face aux conseils municipaux. Elles bénéficient de possibilités de financement plus importantes, tout en devant se soumettre à leur avis pour l'adoption des plans en dernière instance. Le maire de Žergetal le souligne :

Avant, l'AUP travaillait de son côté. Au bout de deux ans, on a décidé que ce mode de fonctionnement n'était pas possible. [...] ils doivent être sous notre autorité. (20 juin 2013)

Les municipalités ont une autre vision de l'environnement : elles envisagent les pâturages comme des ressources à valoriser pour assurer le bien-être des communautés. La loi n'encadre pas les

22 En 2010, les « évènements de Och » ont, selon les sources officielles, causé la mort de 381 personnes.

${ }^{23}$ Ce débat reflète celui existant dans le monde académique [Ellis et Swift 1988 ; Fernandez-Gimenez et Allen-Diaz 1999 ; Isakov et Thorsson 2015]. 
montants donnés par les AUP à la commune : par exemple 30\% du budget à Emgek-Talaa, qui ont servi à répondre à des besoins sociaux pour les ménages les plus pauvres.

Les municipalités sont très influencées par la politique définie par le ministère de l'Agriculture, qui donne la priorité aux opérations de reconstruction et de maintenance des infrastructures d'accès.

Elles incitent donc les AUP à renforcer leurs actions dans ce domaine, au détriment des mesures de suivi ou de protection des ressources. On assiste donc à une convergence des justifications : la réparation et l'entretien des infrastructures, en facilitant le déplacement des bergers et des troupeaux, permettent de limiter la dégradation des pâturages de proximité [Robinson 2016].

\subsection{Les AUP face aux enjeux de coordination entre gestionnaires et usagers}

La création des AUP est intervenue avec le soutien des organisations internationales, qui y ont vu un outil de développement durable. Les diverses approches de l'environnement des organisations gouvernementales ne s'accordent que partiellement avec les objectifs assignés aux nouvelles institutions locales de gestion des pâturages. Ces difficultés se répercutent sur les capacités de ces associations à coordonner leurs actions avec les autres gestionnaires des pâturages et avec les usagers.

Dans les années suivant l'indépendance, les municipalités rurales dans l'incapacité de payer l'impôt foncier ont transféré une partie de leurs pâturages vers les UGF [Steimann 2011]. Le secteur forestier présente aujourd'hui quatre caractéristiques : une très forte centralisation des prises de décision ; un manque de moyens entraînant des réductions de personnel ; une dépendance aux financements extérieurs et l'absence d'information sur les problèmes sociaux rencontrés par les usagers ${ }^{24}$. Les UGF sont enfermées dans un paradoxe. D'une part, elles considèrent que les activités d'élevage mettent en danger les écosystèmes et prônent donc un contrôle important de l'activité pastorale. D'autre part, elles dépendent financièrement des revenus liés à la location des terrains non boisés pour l'élevage ${ }^{25}$, au paiement de l'accès à l'eau pour abreuver le bétail et pour le prélèvement de bois de chauffage ${ }^{26}$.

Les AUP ont hérité de l'approche soviétique de la gestion des pâturages telle qu'elle était portée par les kolkhozes et les sovkhozes. Elles optent pour une organisation territorialisée délimitée par des frontières. Cela se traduit par la volonté de conclure des accords avec les institutions plutôt qu'avec des personnes. Les conflits les plus fréquents opposent les AUP et les usagers qui font paître leur bétail sur les terrains d'une autre commune que la leur en été et refusent de s'acquitter du paiement annuel auprès des deux institutions. Afin d'éviter ces plaintes, les gestionnaires tentent de mettre en place des procédés de transferts de fonds, de sorte que chaque usager paie uniquement I'AUP de sa commune, qui ensuite effectue une redistribution aux AUP dont les terres sont utilisées.

${ }^{24}$ R. Fisher, et al., Poverty and forestry: a case study of Kyrgyzstan with reference to other countries in West and Central Asia, Livelihood Support Programme, Working Paper 13, Food and Agriculture Organization of the United Nations, 2004.

25 Nous n'abordons pas le cas spécifique de la pâture en zones boisées, interdite et néanmoins pratiquée [Borchardt et al. 2011], car il est absent de la zone d'étude.

${ }^{26}$ Voir I. Mestre, A. Ibraimova et B. Azhibekov, Conflicts over pasture resources in the Kyrgyz Republic, rapport de recherche, CAMP Alatoo, Acted, Bichkek, 2013. 
L'utilisation conjointe de pâturages sous l'autorité des AUP et des UGF est également source de tensions. Jusqu'à récemment, les UGF louaient leurs pâturages à des personnes qui s'acquittaient en plus d'une cotisation auprès de leur AUP. Avec le soutien d'ONG, certaines AUP ont tenté de louer des terres aux UGF pour ensuite collecter le paiement pour l'usage des pâturages auprès des habitants, de manière à instaurer un paiement unique à l'AUP. Cependant, dans la majorité des cas, ces accords n'ont pas été reconduits ${ }^{27}$. En effet, les UGF promeuvent une stratégie de conservation basée sur les savoirs experts et une gestion centralisée. Elle se concrétise par un encadrement des pratiques agropastorales et un suivi de l'état des ressources naturelles plus stricts. Bénéficiant rarement de soutien technique de la part de spécialistes, les AUP sont perçues comme incompétentes par l'Agence de protection des écosystèmes, qui ne reconnaît pas les savoirs locaux. Par ailleurs, comme mentionné ci-dessus, l'Agence et les UGF tirent une grande partie de leurs revenus de la location de terre à des fins de pâture et, craignant de ne pas recevoir les sommes dues, exigent des prépaiements que les AUP ne peuvent fournir.

Bien que la loi « Sur les pâturages » traite en premier lieu de l'élevage agropastoral, les plans de gestion et d'utilisation incluent d'autres usages. Un amendement (arrêté $n^{\circ} 515,13 / 09 / 2013$ ) prévoit que l'utilisation des ressources pastorales à des fins autres que l'élevage doit respecter les principes de transparence, de continuité, d'accessibilité, de rationalité et de durabilité, sans fournir plus d'éléments opérationnels. Les AUP ont du mal à faire respecter les obligations de paiement prises par les usagers. En effet, elles n'ont pas les connaissances réglementaires relatives aux activités non agricoles ni les moyens d'assurer un suivi géobotanique pour évaluer leur impact sur les ressources pastorales et estimer le montant des dédommagements. Dans la pratique, les AUP décident de manière autonome des modes de calcul pour les paiements qu'elles justifient selon différentes approches de l'environnement. Nous en donnerons trois exemples : l'accueil de touristes en yourtes, les activités minières industrielles et l'apiculture.

Les habitants qui accueillent des touristes dans des yourtes situées dans les pâturages estivaux doivent acquitter à l'AUP une contribution aux activités communautaires pour l'utilisation des infrastructures d'accès. Leurs revenus étant plus importants que ceux des ménages menant uniquement des activités d'élevage, l'AUP justifie ce paiement par une répartition équitable des bénéfices du tourisme entre tous les ménages. Elle peut ainsi allouer ces sommes à des routes et à des ponts que tous peuvent emprunter. Cette stratégie s'appuie sur la combinaison de deux approches. Premièrement, celle du ministère de l'Agriculture centrée sur les infrastructures et, deuxièmement, celle des municipalités, pour qui la gestion des pâturages doit être un moyen au service de l'équité, afin que tous les ménages exercent une activité leur permettant de vivre dignement.

Le calcul du paiement des exploitations minières aux AUP se justifie également par la répartition équitable des revenus générés. II ne tient pas compte de l'absence d'impact direct sur les activités pastorales, les exploitations minières étant en général situées à un étage altitudinal plus élevé, ni du bénéfice tiré par les éleveurs-bergers et les bergers des infrastructures d'accès construites par les entreprises minières. Le montant de cette contribution des entreprises minières aux AUP génère des conflits [Doolot et Heathershaw 2015].

${ }^{27}$ A. Ibraimova, et al., Conflicts over Pastures Resources and Ways Forward, Bishkek, Camp Alatoo, 2015 (<https://pasture.klink.asia/klink/c5d2dd/document>). 
Enfin le cas de l'apiculture manifeste l'opposition entre les approches. Dans un premier temps, les AUP ont demandé une contribution aux apiculteurs pour l'usage des routes et des ponts. Ces derniers, avec le soutien des organisations internationales, ont alors fait reconnaître les bénéfices environnementaux de la pollinisation pour en être exemptés. S'appuyant sur le concept de développement durable, ils ont souligné l'impact positif du travail des abeilles sur le couvert végétal et l'élevage.

\subsection{Conclusion}

Des approches plurielles de l'environnement orientent les stratégies des acteurs et influencent le fonctionnement des associations d'usagers des pâturages. Selon la notion de l'environnement qu'elles adoptent, les AUP privilégient soit l'amélioration des infrastructures d'accès aux pâturages, comme c'est le cas des Associations d'usagers de l'eau [Schmitt 2015], soit un mode de gestion où la protection des ressources naturelles est aussi importante que le développement et le maintien des infrastructures. L'absence d'un cadre accepté par tous les partenaires pour l'évaluation des AUP [Dörre 2015], incite à renouveler l'appel de A. Agrawal et E. Ostrom pour « commencer le long et ardu processus de réflexion sur une mesure de la performance environnementale qui ne fasse pas des changements locaux de simples épiphénomènes » [op. cit. : 64 ${ }^{28}$.

Cependant, si les objectifs des AUP peinent à être clarifiés en raison du flou qui entoure la notion d'environnement, leur capacité à s'approprier les mécanismes pour peser sur les acteurs de l'échelle nationale montre la capacité d'action des acteurs locaux dans le nouveau mode de gestion. Enfin, si la diversité des approches environnementales freine l'application d'une politique unique pour la décentralisation de la gestion des pâturages, elle offre à ces associations la possibilité de les mobiliser de manière flexible en fonction des objectifs poursuivis, ce qui leur permet une grande souplesse dans la mise en place des mesures et dans la définition de leurs relations avec les autres acteurs locaux.

\section{Bibliographie}

Agrawal, Arun et Elinor Ostrom, 2007, "Decentralization and Community-Based Forestry: Learning from Experience ", in E. L. Webb et G. Shivakoti, Decentralization, Forests and Rural Communities: Policy Outcomes in South and South East Asia. New Dehli, Sage Publishing : 44-67.

Agrawal, Arun et Jesse RiBot, 1999, "Accountability in Decentralization: A Framework with South Asian and West African Cases ", The Journal of Developing Areas 33 (4) : 473-502.

Borchard, Peter, et al., 2011, "Mountain Pastures and Grasslands in the SW Tien Shan, Kyrgyzstan - Floristic Patterns, Environmental Gradients, Phytogeography, and Grazing Impact ", Journal of Mountain Science 8 (3) : 363-373.

CREWETt, Wibke, 2012, «Improving the Sustainability of Pasture Use in Kyrgyzstan. The Impact of Pasture Governance Reforms on Livestock Migration ", Mountain Research and Development 32 (3) : 267-274. - 2016, Decentralization in the Kyrgyz agricultural sector. An institutional analysis. PhD Thesis, Humbolt University, Berlin.

28 Notre traduction. 
Doolot, Asel et John Heathershaw, 2015, "State as Resource, Mediator and Performer: Understanding the Local and Global Politics of Gold Mining in Kyrgyzstan », Central Asian Survey 34 (1) : 93-109.

DörRE, Andrei, 2015, «Promises and realities of community-based pasture management approaches: Observations from Kyrgyzstan ", Pastoralism: Research, Policy and Practice 5 : 15.

EluIS James E. et David M. SWIFT, 1988, "Stability of African pastoral ecosystems: alternate paradigms and implications for development ", Rangeland Ecology \& Management/Journal of Range Management Archives 41: 450-459.

Fernandez-Gimenez, Maria E. et Barbara Allen-Diaz, 1999, « Testing a non equilibrium model of rangeland vegetation dynamics in Mongolia ", Journal of Applied Ecology 36: 871-885.

Gumuchian, Hervé et al., 2003, Les acteurs, ces oubliés du territoire. Paris, Anthropos.

ISAeVA, Aiganysh et Jyldyz Shigaeva, 2017, "Soviet Legacy in the Operation of Pasture Governance Institutions in Present-Day Kyrgyzstan », Journal of Alpine Research. Revue de géographie alpine 105 (1) (<https://rga.revues.org/3631>).

Isakov Azamat et Johann Thorsson, 2015, Assessment of the land condition in the Kyrgyz Republic with respect to grazing and a possible development of a quoting system on the local governmental level. Bichkek, V.R.S. Company Ltd (<http://naturalresources-

centralasia.org/flermoneca/assets/files/Assessment\%20of\%20the\%20land\%20cond ition\%20in\%20the\%20KR\%20with\%20respect\%20to\%20grazing..._EN.pdf>).

LEVINE, Jordan, et al., 2017, " A cognitive approach to the post-Soviet Central Asian pasture puzzle: new data from Kyrgyzstan », Regional Environmental Change 17 (3) : 94-947.

MeSTRE, Irène, 2017, "Quand les bergers creusent la montagne. Impact des activités minières artisanales sur les systèmes agropastoraux du Kirghizstan. Étude de cas dans la région de Naryn ", Journal of Alpine Research. Revue de géographie alpine 105 (1) (<https://rga.revues.org/3575>).

Ostrom, Elinor, 1990, Governing the Commons: the Evolution of Institutions for Collective Action. Cambridge, Cambridge University Press.

Petric, Boris-Mathieu, et al., 2004, "L'émergence de nouveaux pouvoirs locaux sur les cendres d'un kolkhoze kirghize (oblast de Naryn) ", Cahiers d'Asie centrale 13/14: 21-44.

Robinson, Sarah, 2016, "Land Degradation in Central Asia: Evidence, Perception and Policy " in R. Behnke et M. Mortimore (dir.), The End of Desertification?, Heidelberg; Springer: 451-490.

Shigaeva, Jyldyz, Bettina Wolfgramm et Chad Dear, 2013, Sustainable Land Management in Kyrgyzstan and Tajikistan: A Research Review. Bishkek, Mountain Societies Research Institute, University of Central Asia. 
ShigaeVA, Jyldyz, et al., 2016, "Decentralizing Governance of Agropastoral Systems in Kyrgyzstan: An Assessment of Recent Pasture Reforms", Mountain Research and Development 36 (1): 91-101.

SCHMIDT, Matthias et Andrei DoerRe, 2011, "Changing Meanings of Kyrgyzstan's nut Forests from Colonial to Post-Soviet Times ", Area 43 (3) : 288-296.

SCHMITT, Florian, 2015 "'Functional' governance as an alternative to 'territorial' governance? The case of the Kyrgyz irrigation sector and the implementation of Water User Associations (WUAs)", Crossroads Asia Working Paper Series 30 (<https://bonndoc.ulb.unibonn.de/xmlui/bitstream/handle/20.500.11811/165/Crossroads_WP_30_Schmitt_ Functional_Governance.pdf?sequence $=1$ \&isAllowed $=\mathrm{y}>$ )

SteimanN, Bernd, 2011, Making a living in uncertainty: agro-pastoral livelihoods and institutional transformations in post-socialist rural Kyrgyzstan. Thèse de doctorat en géographie. Zurich, Université de Zurich.

Undeland, Asyl et Andrew M. Mitchell, 2015, « Kyrgyz Republic - Communities forests and pastures ", working paper.

WeIneR, Douglas R., 1982, "The Historical Origins of Soviet Environmentalism ", Environmental Review: ER 6 (2) : 42-62.

Wolfgramm, Bettina, Jyldyz Shigaeva et Chad Dear, 2015, "The Research-Action Interface in Sustainable Land Management in Kyrgyzstan and Tajikistan: Challenges and Recommendations ", Land Degradation \& Development 26 (5) : 480-490.

Žaparov, Amantur, 2010, "L'élevage du cheval au Kirghizstan », trad. du russe par Carole Ferret, Études mongoles et sibériennes, centrasiatiques et tibétaines, 41 (<https://emscat.revues.org/1575\#quotation>).

Cet article analyse les effets de la diversité des conceptions de l'environnement dans un processus de décentralisation de la gestion des pâturages en s'appuyant sur le cadre conceptuel de la décentralisation de la gestion des ressources naturelles développé par Arun Agrawal et Elinor Ostrom. Au Kirghizstan, la mise en place en place d'institutions de gestion communautaire des pâturages à l'échelle locale, les Associations d'usagers des pâturages, a fait émerger l'opposition entre les conceptions de l'environnement portées par les acteurs gouvernementaux, non gouvernementaux et internationaux. À l'échelle locale, les Associations d'usagers des pâturages s'approprient ces approches de manière sélective pour définir les relations avec les usagers non agropastoraux et s'organisent pour influencer les acteurs de l'échelle nationale.

Mots clés : Kirghizstan, agro-pastoralisme, approches de l'environnement, gestion communautaire des ressources naturelles, décentralisation.

This article aims to analyse the effects of the diversity of the concepts of environment in the pasture management decentralisation process. In this respect, we use the conceptual framework developed by Agrawal and 
Ostrom. In Kyrgyzstan, the implementation of local community-based institutions for pasture management reinvigorated the discrepancies between the concepts of environment of the governmental, nongovernmental and international stakeholders. At the local level, pasture user associations appropriate these concepts selectively in order to define their interactions with non-agropastoral users and organize themselves to influence national-level stakeholders.

Keywords: Kirghizstan agro-pastoralism, concept of environment, communitybased natural resource management, decentralization 